\title{
Range-Measurement Sensor to Improve the Authentication Workflow for Users of a Hospital Information System: A Proof-of- Concept Study
}

\author{
Kei TERAMOTO ${ }^{\mathrm{a}, 1}$, Shigeki KUWATA ${ }^{\mathrm{b}}$, Masashi HAMAMOTO ${ }^{\mathrm{c}}$ and Hiroshi \\ $\mathrm{KONDOH}^{\mathrm{a}}$ \\ ${ }^{a}$ Division of Medical Informatics, Tottori University Hospital, Japan \\ ${ }^{\mathrm{b}}$ Department of Clinical Information Management, Nara City Hospital, Japan \\ ${ }^{\mathrm{c}}$ Ricoh Japan Co., Ltd
}

\begin{abstract}
In this study, we developed an authentication rangefinder (AR) system for hospital information system (HIS) terminals to support the user authentication workflows. The logoff process of the AR system is triggered if no object is placed at least $90 \mathrm{~cm}$ in front of the HIS terminal laptop for $\geq 5 \mathrm{~s}$. We conducted an anonymous survey of medical staff who used the AR system. 33/42(78\%) respondents acknowledged an improvement in the logoff process. This study indicates that the AR system improves the user authentication workflow.
\end{abstract}

Keywords. Authentication system, hospital information system, medical staff

\section{Introduction}

Medical staff assigned to wards frequently log in and log off from hospital information system (HIS) terminals as they alternate between treating patients and referring to recording in patients' electronic medical records [1-3]. We surveyed the average number of logins per day made by doctors and nurses at Tottori University Hospital and found that doctors and nurses logged in 22 and 41 times, respectively. If the authentication process takes a long time, operational efficiency can be significantly reduced. If staff do not log out of terminals to save the time and effort of the HIS authentication process, not only is there an increased risk of data breaches resulting from shoulder surfing by third party applications and unauthorized logins but the operational efficiency of HIS terminals will also decrease. Therefore, simplifying login and logoff for HIS terminals is very important for the staff of medical institutions that have implemented HIS to improve workflow efficiency [4]. This study was aimed at developing an authentication rangefinder system (AR system) for to achieve an efficient HIS-terminal authentication workflow required by medical staff, and it was evaluated using a user survey.

${ }^{1}$ Corresponding Author, Kei Teramoto, Division of Medical Informatics, Tottori University Hospital, 36-1 Nishi-cho, Yonago, Tottori, 683-8504, Japan; E-mail: kei@tottori-u.ac.jp. 


\section{Method}

We describe a method for authorizing login and logoff of HIS terminals by using an AR system. Login authentication using the AR system identifies users according to two elements: the BLE staff card worn by the user and a vein biometric authentication device. BLE staff authentication is equipped with a function that transmits a beacon signal to the staff ID cards, by which the Bluetooth functionality of the HIS terminal detects that a user is in the vicinity of a PC. The laser rangefinder module was detected when the medical staff are at least $90 \mathrm{~cm}$ away from the HIS terminal, and if $\geq 5 \mathrm{~s}$ have passed, the screen saver screen is activated. The screen saver was configured to be canceled if the user returns to the $\mathrm{PC}$ within $30 \mathrm{~min}$. If a beacon signal cannot be detected for at least $30 \mathrm{~min}$, the system will log off. The AR system was introduced into a general ward of Tottori University Hospital for one year. After that, the system was evaluated by the nurse using the paper-based questionnaire.

\section{Results}

For the login process using the AR system, 21 of 44 respondents acknowledged an improvement in the workflow; 16 were indifferent, while 7 responded that the workflow was worse. 5 of 7 answered that they experienced frequent login failures. 33 of 42 responded that the AR system improved the logoff process workflow. Negative feedback concerning operational improvements was that the screen saver did not disappear promptly and the distance required before the screen cleared felt long; this could have been caused by the values set for the rangefinder.

\section{Discussion}

The AR system was developed to support HIS authentication workflows and simplify the sequence of HIS terminal operations from login to logoff. In the login process, the accuracy of login authentication was an issue, with only $47.7 \%$ of respondents feeling that the workflow was improved. The logoff process could be further improved by adjusting the settings for the startup time of the screen saver and the laser rangefinder distance. The introduction of the AR system could help medical institutions improve the security of user authentication as well as the efficiency of their operations.

\section{References}

[1] Andersen P, Lindgaard AM, Prgomet M, Creswick N, Westbrook JI. Mobile and fixed computer use by doctors and nurses on hospital wards: multi-method study on the relationships between clinician role, clinical task, and device choice. J Med Internet Res. 2009;11(3):e32.

[2] Pearce C, Walker H, O'Shea C. A visual study of computers on doctors' desks. Inform Prim Care. 2008;16(2):111-7.

[3] Badger SL, Bosch RG, Toteja P. Rapid implementation of an electronic health record in an academic setting. J Healthc Inf Manag. 2005;19(2):34-40.

[4] Fontaine J, Zheng K, Van De Ven C, Li H, Hiner J, Mitchell K, et al. Evaluation of a proximity card authentication system for health care settings. Int J Med Inform. 2016; 92:1-7. 